\title{
Supplier selection and consistency level measurement of decision maker using AHP method and expertise level model
}

\author{
Fatkhor Rozi $^{1, *}$ and Evy Herowati ${ }^{1}$ \\ ${ }^{1}$ Departement of Industrial Engineering, University of Surabaya, 60293 Surabaya, Indonesia
}

\begin{abstract}
Raw materials are basic need for manufacturing companies to facilitate the production process. Therefore, supplier selection process is an important process for the company, because by choosing the right suppliers of raw materials will benefit the company. To choose the right supplier is also determined by the decision taken by the decision maker (DM). In addition, the weight of DM assessment will differ on different criteria because DM experts in a particular field are not necessarily experts in other fields. Therefore, in this paper is discussed about the measurement of the weight of each criterion and alternative to choose the best supplier alternative using AHP method. In addition, this paper also measures the consistency of DM in making decisions using expertise level models.
\end{abstract}

\section{Introduction}

Raw materials are basic requirement for manufacturing companies to facilitate the production process. The late delivery of raw materials resulted in the production process in the company becoming obstructed. This results in other problems that arise, such as unmet demand, and customer disappointment. Therefore, the selection of raw material suppliers is the key for manufacturing companies in running their business. In selecting raw material suppliers, the criteria considered are qualitative and quantitative and are MCDMs because more than one criterion is considered. Criteria to consider include quality, price, capacity, delivery, and service.

The supplier selection model can be divided into single sourcing and multiple sourcing [1]. Single sourcing is choosing one of the existing supplier alternatives based on the best ranking. Meanwhile, multiple sourcing is choosing more than one supplier because no supplier is able to meet the total demand required or as a procurement strategy. In a company, it usually takes more than a decision maker (DM) in choosing raw material suppliers. This is because the individual skills of a DM are not sufficient to provide an assessment of all aspects of the MCDM problem, since an individual can only judge on the part of the mastered problem [2]. In addition, the weight of the DM assessment will differ on different criteria because an expert DM in a particular field is not necessarily an expert

* corresponding author: fatkhorrozi@gmail.com 
in another field [3]. This indicates more than one DM is required in decision making. In addition, expert decision making can improve the quality of the decision.

In this paper, the method used to determine the weight of each criterion and the alternative is the AHP method. The AHP method has been often applied as one of the methods in decision making. In addition, this method is considered easy to apply and is quite accurate. In addition, in this paper is also measured consistency DM in making decisions using expertise level model. The pairwise comparison data used in the weighting of each criterion and the alternative will be transformed into Fuzzy Preference Relations (FPR) scale $[0 \ldots 1]$. For the next process is done additive consistency before the process of expertise level. This is done to find out how big the inconsistency of DM in decision making. In addition, the results of this ranking can also be used as an evaluation of management in determining the people who act as DM later.

\section{Literature Review}

\subsection{AHP}

Analytic Hierarchy Process (AHP) method is decision support model developed by Thomas L. Saaty to find the ranking or priority order of various alternatives that exist in solving a problem. In complex situations, decision-making is not influenced by only one criterion but rather multi criteria.

Basically the AHP method is measurement theory used to find the interval scale of pairwise comparisons. The AHP method is used to examine the problems that begin by defining the problem and then compiling it into the hierarchy. According to Saaty, there are several basic principles to solve problems with the AHP method, among others: Decomposition, Comparative Judgment, Synthesis of Priority, and Logical Consistency [4].

\subsection{The Transformation From MPR to FPR}

The transformation of the Multiplicative Preference Ratio (MPR) which has a ratio of [1/9, 9] to FPR which has the value [0,1] is as follows [7].

$$
P_{i j}=0.5\left(1+\log _{9} a_{i j}\right)
$$

\subsection{The Expert's Expertise Level}

This method assesses that the quality of assessment of DM can be seen from the DM's ability to repeat their judgments consistently and to be able to distinguish between similar but unequal cases [3, 8]. Consistency assessment of DM can be seen from the value of CWS-Index (Ratio Cochran Weiss Shanteau), i.e. the ratio between discrimination and inconsistency values as in equations (2), (3), and (4).

$$
\begin{aligned}
\text { CWS-Index } & =\frac{\text { Discrimination }}{\text { Inconsistency }} \\
\text { Discrimination } & =\sqrt{\frac{\sum_{j=1}^{n} r(M j-G M)^{2}}{n-1}} \\
\text { Inconsistency } & =\sqrt{\frac{\sum_{j=1}^{n} \sum_{i=1}^{r} r(M i j-G M)^{2}}{n(r-1)}}
\end{aligned}
$$


Where:

$r \quad:$ : The number of replications

$M_{j} \quad:$ The average of individual values for case-j

GM : Grand mean of all individual values

$n \quad:$ : The number of different cases

$M_{i j} \quad:$ The individual value for replication-i case-j

If the CWS-ratio Ratio of DM is higher, also the higher the expertise level of DM [3].

\subsection{The FPR's AC property}

FPR is a method often used in GDM (Group Decision Making) and has the reciprocity consistency $p_{i j}+p_{j i}=1$. AC (Additive Consistency) is proposed as a condition of consistency of an FPR $[5,6]$. Where each element of the matrix $p=p_{i k}$ is estimated based on the corresponding FPR obtained from the DM assessment of the alternative decision using three formulas [9].

$$
\begin{aligned}
& \varepsilon p_{i k}^{j 1}=p_{i j}+p_{j k}-\frac{1}{2}, j \neq i, k \\
& \varepsilon p_{i k}^{j 2}=p_{j k}-p_{j i}+\frac{1}{2}, j \neq i, k \\
& \varepsilon p_{i k}^{j 3}=p_{i j}-p_{k j}+\frac{1}{2}, j \neq i, k
\end{aligned}
$$

Where:

$\varepsilon p_{i k}^{j 1}$ : Estimation of $p_{i k}$ using the first formula.

$\varepsilon p_{i k}^{j 2}$ : Estimation of $p_{i k}$ using the second formula.

$\varepsilon p_{i k}^{j 3}$ : Estimation of $p_{i k}$ using the third formula.

This estimate allows the nature of the AC to be used to measure the consistency level of DMs based on their assessment [3].

\subsection{The OWA Operator}

The principle of determining the weight of DM assessment is based on the principle of determining the weight of OWA operators. The weight of the OWA operator is introduced by Yager [10] which uses the number $r$ in determining the weight of the OWA operator and uses a special form of the Basic Unit Monotone Increasing Function (BUM) which is Q $(r)$ $=r^{\alpha}$. Q $(r)$ is an accumulation weight function which has an interval range $[0,1]$, so that the total weight can be obtained is 1 [11]. The OWA operator is aggregation operator in which input arguments are sorted from the largest to the smallest.

\section{The Proposed Model}

In this paper, the AHP model is used to calculate the weights of each of the existing criteria and alternatives. So it will get the best supplier alternative that will supply raw materials in the company. In addition, to measure the consistency of each DM, the ratio of CWS-Ratio 
(Cochran Weiss Shanteau ratio) is performed, ie. the ratio between discrimination and inconsistency values as in equations (2), (3), and (4). In addition, there is also QGDD measurement to determine the best alternative decision dominance against other alternatives.

\section{Illustrative Example}

There are $3 \mathrm{DM}$ in charge of assessing suppliers (A1, A2, and A3) to be selected to supply raw materials in the company. Criteria considered in this decision-making are price (K1), capacity (K2), quality (K3), delivery (K4), and service (K5). The combined results from the weight assessment of each criterion of all DM are shown as in table (1).

Table 1. Comparison matrice for criteria.

\begin{tabular}{|l|c|c|c|c|c|}
\hline & K1 & K2 & K3 & K4 & K5 \\
\hline K1 & 1.00 & 3.00 & 2.67 & 0.58 & 3.67 \\
\hline K2 & 0.33 & 1.00 & 2.33 & 0.33 & 2.00 \\
\hline K3 & 0.38 & 0.43 & 1.00 & 0.30 & 2.33 \\
\hline K4 & 1.71 & 3.00 & 3.30 & 1.00 & 5.00 \\
\hline K5 & 0.27 & 0.50 & 0.43 & 0.20 & 1.00 \\
\hline
\end{tabular}

While the weight assessment results for "Price" criteria of all DMs for all alternatives are shown as in tables (2).

Table 2. Pairwise comparisson for "Price (K1)" criteria of each DM.

\begin{tabular}{|c|c|c|c|}
\hline & \multicolumn{3}{|c|}{ DM-1 } \\
\hline K1 & A1 & A2 & A3 \\
\hline A1 & 1 & 3 & 1 \\
\hline A2 & 0.33 & 1 & 0.5 \\
\hline A3 & 1 & 2 & 1 \\
\hline & \multicolumn{3}{|c|}{ DM-2 } \\
\hline K1 & A1 & A2 & A3 \\
\hline A1 & 1 & 5 & 1 \\
\hline A2 & 0.2 & 1 & 0.33 \\
\hline A3 & 1 & 3 & 1 \\
\hline & \multicolumn{3}{|c|}{ DM-1 } \\
\hline K1 & A1 & A2 & A3 \\
\hline A1 & 1 & 2 & 1 \\
\hline A2 & 0.5 & 1 & 0.25 \\
\hline A3 & 1 & 4 & 1 \\
\hline
\end{tabular}

Table 3. Aggregated results for each alternative according to each criteria.

\begin{tabular}{|l|c|c|c|c|}
\hline & Weight & A1 & A2 & A3 \\
\hline K1 & 0.285 & 0.43 & 0.15 & 0.42 \\
\hline K2 & 0.147 & 0.49 & 0.21 & 0.30 \\
\hline K3 & 0.110 & 0.56 & 0.13 & 0.31 \\
\hline K4 & 0.390 & 0.57 & 0.17 & 0.26 \\
\hline K5 & 0.067 & 0.57 & 0.23 & 0.19 \\
\hline \multirow{4}{*}{ Total } & 0.51 & 0.17 & 0.32 \\
\cline { 2 - 5 }
\end{tabular}

From the result of measurement of each supplier's weight and alternative, then the result is obtained as in table (3). Alternative A1 has the best ranking compared to other supplier 
alternatives based on the choice of all DMs if using AHP method. Then to measure the consistency of the DM decision, it is measured Ratio - CWS (Cochran Weiss Shanteau ratio). The estimated value is derived from the pairwise comparison value of each criterion against the available alternatives. To perform the transformation from MPR to FPR is used equation (1) and the result as shown in table (4).

Table 4. Transformation from MPR to FPR for "Price (K1)" Criteria of DM-1.

\begin{tabular}{|c|c|c|c|c|c|c|c|c|}
\hline K1 & $\mathrm{A} 1$ & A2 & A3 & \multirow{4}{*}{ to } & K1 & $\mathrm{A} 1$ & A2 & A3 \\
\hline A1 & 1.00 & 3.00 & 1.00 & & A1 & 0.50 & 0.75 & 0.50 \\
\hline $\mathrm{A} 2$ & 0.33 & 1.00 & 0.50 & & A2 & 0.25 & 0.50 & 0.34 \\
\hline A3 & 1.00 & 2.00 & 1.00 & & A3 & 0.50 & 0.66 & 0.50 \\
\hline
\end{tabular}

$\mathrm{a} 11=0.5\left(1+\log _{9} 1\right)=0.5$

$\mathrm{a} 12=0.5\left(1+\log _{9} 3\right)=0.75$

Then, the estimated value is calculated using the $\mathrm{AC}$ formula as performed in the previous study [9].

Table 5. Estimation and calculation for "Price" criteria of DM-1.

\begin{tabular}{|c|c|c|c|c|c|}
\hline & $\mathrm{OA}$ & F.1 & F.2 & F.3 & $M j$ \\
\hline$p 12$ & 0.75 & 0.66 & 0.66 & 0.66 & 0.68 \\
\hline$p 13$ & 0.50 & 0.59 & 0.59 & 0.59 & 0.57 \\
\hline$p 21$ & 0.25 & 0.34 & 0.34 & 0.34 & 0.32 \\
\hline$p 23$ & 0.34 & 0.25 & 0.25 & 0.25 & 0.27 \\
\hline$p 31$ & 0.50 & 0.41 & 0.41 & 0.41 & 0.43 \\
\hline p32 & 0.66 & 0.75 & 0.75 & 0.75 & 0.73 \\
\hline & \multicolumn{2}{|c|}{$r(M j-G M)^{2}$} & \multicolumn{3}{|c|}{$\sum_{i=1}^{r} r(M i j-G M)^{2}$} \\
\hline$p 12$ & \multicolumn{2}{|c|}{0.098} & \multicolumn{3}{|c|}{$2 \mathrm{E}-03$} \\
\hline$p 13$ & \multicolumn{2}{|c|}{0.014} & \multicolumn{3}{|c|}{$2 \mathrm{E}-03$} \\
\hline$p 21$ & \multicolumn{2}{|c|}{0.098} & \multicolumn{3}{|c|}{ 2E-03 } \\
\hline$p 23$ & \multicolumn{2}{|c|}{0.154} & \multicolumn{3}{|c|}{$2 \mathrm{E}-03$} \\
\hline$p 31$ & \multicolumn{2}{|c|}{0.014} & \multicolumn{3}{|c|}{$2 \mathrm{E}-03$} \\
\hline$p 32$ & \multicolumn{2}{|c|}{0.154} & \multicolumn{3}{|c|}{$2 \mathrm{E}-03$} \\
\hline & \multicolumn{2}{|c|}{0.53} & \multicolumn{3}{|c|}{0.010} \\
\hline
\end{tabular}

From the results shown as in table (5), then the calculation can be obtained as equations (2), (3), and (4). The values of CWS-Index for "Price" criteria from each DM can be seen as in table (6). Based on these results, the DM-2 has the best expertise rating for "price" criteria because it has the largest CWS-Index value (13.736) when compared with CWS-Index values from others DM.

Discrimination $=\sqrt{\frac{\sum_{j=1}^{n} r(M j-G M)^{2}}{n-1}}=\sqrt{\frac{0.53}{(6-1)}}=0.327$

Inconsistency $=\sqrt{\frac{\sum_{j=1}^{n} \sum_{i=1}^{r} r(M i j-G M)^{2}}{n(r-1)}}=\sqrt{\frac{0.01}{6 x(3-1)}}=0.028$ 
CWS-Index for DM-1 $=\frac{\text { Discrimination }}{\text { Inconsistency }}=\frac{0.327}{0.028}=11.566$

Table 6. Discrimination, inconsistency, and CWS-Index for "Price" criteria of each DM.

\begin{tabular}{|l|c|c|c|}
\hline & DM-1 & DM-2 & DM-3 \\
\hline Discrimination & 0.327 & 0.489 & 0.394 \\
\hline Inconsistency & 0.028 & 0.036 & 0.048 \\
\hline CWS-Index & 11.566 & 13.736 & 8.149 \\
\hline
\end{tabular}

After obtaining the CWS index of each DM, we can calculate the weight of each DM for "price (K1)" criteria as shown in table (7). From the calculation of weight assessment for the price criteria for DM-1 is $34.16 \%$, DM-2 is $36.56 \%$, and DM-3 is $29.28 \%$.

Table 7. Weight of the DM assessment for "Price" criteria.

\begin{tabular}{|l|r|r|r|}
\hline & \multicolumn{1}{|c|}{ DM-2 } & \multicolumn{1}{c|}{ DM-1 } & \multicolumn{1}{c|}{ DM-3 } \\
\hline CWS- Index & 13.736 & 11.566 & 8.149 \\
\hline Log(CWS-Index) & 1.138 & 1.063 & 0.911 \\
\hline $\begin{array}{l}\text { Accumulated } \\
\text { (Log(CWS-Index)) }\end{array}$ & 1.138 & 2.201 & 3.112 \\
\hline $\begin{array}{l}\text { r=Normalized(Accumulated } \\
\text { (Log(CWS-Index)) }\end{array}$ & 0.366 & 0.707 & 1 \\
\hline Q(r) $=\mathrm{r}^{1}$ & 0.366 & 0.707 & 1 \\
\hline Importance Weights (\%) & 36.562 & 34.163 & 29.275 \\
\hline
\end{tabular}

Table 8. Combined FPR value for "Price" criteria.

\begin{tabular}{|l|c|c|c|}
\hline Price & A1 & A2 & A3 \\
\hline A1 & 0.50 & 0.77 & 0.50 \\
\hline A2 & 0.23 & 0.50 & 0.26 \\
\hline A3 & 0.50 & 0.74 & 0.50 \\
\hline
\end{tabular}

After obtaining the combined FPR value of each criterion, the next step is to calculate the total combined FPR value. The total combined FPR value as shown in table (10) is derived from multiplying the value in the matrix element of each criterion by the weight of each criterion. The weight of each criterion is determined by the owner of the company because it is understood to be the criterion considered important in decision making as shown in table (9).

Table 9. Weights for each criteria.

\begin{tabular}{|c|c|}
\hline $\mathrm{K} 1$ & 0.3 \\
\hline $\mathrm{K} 2$ & 0.2 \\
\hline $\mathrm{K} 3$ & 0.15 \\
\hline $\mathrm{K} 4$ & 0.25 \\
\hline $\mathrm{K} 5$ & 0.1 \\
\hline \multicolumn{1}{l}{} & 1 \\
\cline { 2 - 2 }
\end{tabular}

$\mathrm{a} 11=(0.3 * 0.5)+(0.2 * 0.5)+(0.15 * 0.5)+(0.25 * 0.5)+$ $(0.1 * 0.5)=\mathbf{0 . 5 0}$ 
Table 10. Total combined value of FPR.

\begin{tabular}{|c|c|c|c|}
\hline K1 & A1 & A2 & A3 \\
\hline A1 & 0.50 & 0.75 & 0.62 \\
\hline A2 & 0.25 & 0.50 & 0.33 \\
\hline A3 & 0.38 & 0.67 & 0.50 \\
\hline
\end{tabular}

After obtaining the total combined value of FPR, the next step is to sort the combined value of FPR starting from the largest such as the OWA operator principle.

Table 11. QGDD calculation process.

\begin{tabular}{|c|c|c|c|c|c|c|c|}
\hline K1 & A1 & A2 & A3 & \multirow{4}{*}{ to } & A1 & A2 & A3 \\
\hline A1 & 0.50 & 0.75 & 0.62 & & & 0.75 & 0.62 \\
\hline A2 & 0.25 & 0.50 & 0.33 & & 0.25 & & 0.33 \\
\hline A3 & 0.38 & 0.67 & 0.50 & & 0.38 & 0.67 & \\
\hline & & & & & \multicolumn{3}{|c|}{ to } \\
\hline & & & & & 0.75 & 0.62 & \\
\hline & & & & & 0.33 & 0.25 & \\
\hline & & & & & 0.67 & 0.38 & \\
\hline
\end{tabular}

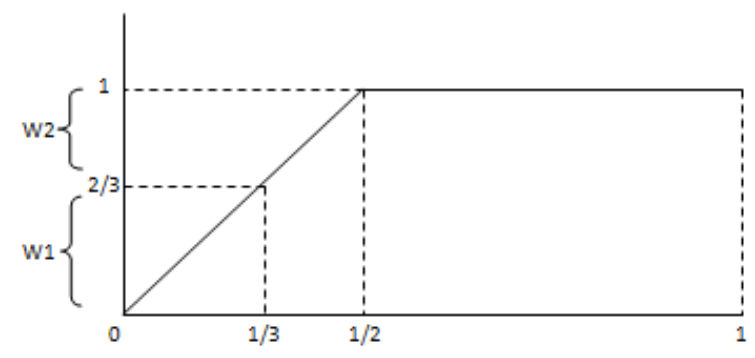

Fig. 1. Fuzzy quantifier “At Least Half” for three alternative.

The weight of the OWA operator is obtained based on the fuzzy quantifier "At Least Half" which has a decision interval $[0,0,5]$, so the weight used for the three alternative decisions is $(2 / 3,1 / 3)$.

$\mathrm{w} 1=2 / 3$

$\mathrm{w} 2=1-(2 / 3)=1 / 3$

$\mathrm{w} 3=0$

After that, QGDD calculations are performed to see the best decision alternative. QGDD serves to measure the dominance of alternative decisions on other alternative decisions on the fuzzy majority as as in equation (8) [12].

QGDDi $=$ Fq (Pij Grup, $\mathrm{j}=1,2, \ldots, \mathrm{n}, \mathrm{j} \neq 1)$ 
Table 12. QGDD calculation result.

\begin{tabular}{|c|c|c|c|c|}
\hline & W1 & W2 & Total & Rank \\
\hline QGDD1 & 0.504 & 0.204 & 0.708 & 1 \\
\hline QGDD2 & 0.222 & 0.082 & 0.303 & 3 \\
\hline QGDD3 & 0.448 & 0.126 & 0.574 & 2 \\
\hline
\end{tabular}

QGDD1 $=(0.667 * 0.504)+(0.333 * 0.204)=0.708$

QGDD1 $=(0.667 * 0.222)+(0.333 * 0.082)=0.303$

QGDD1 $=(0.667 * 0.448)+(0.333 * 0.126)=0.574$

From the calculation of QGDD can be concluded that alternative A1 is an alternative decision that dominates other decision alternatives with the highest degree. In addition it can be concluded that alternative A1 is the best supplier alternative.

\section{Conclusion}

This paper discusses how to measure the weight of each criterion and the alternative using the AHP method. Supplier A1 has the best ratings compared to other supplier alternatives based on the choice of all DMs. The result of AHP method is also similar to QGDD calculation, where alternative A1 has the highest degree compared to other supplier alternatives. In addition, it also measured the consistency of each DM in making decisions using expertise level model. It can be concluded that this model can know the DM which has the best consistency level, and the highest CWS-Index value indicating the level of DM expertise in making decisions for each criterion. So the results can be used as an evaluation material for the company in determining who the decision maker.

\section{References}

1. C. Guo, PhD diss., University of Tennessee (2014)

2. E. N. Weiss, V. R. Rao, Decision Sciences, 18 (1987)

3. E. Herowati, J. Parung, U. Ciptomulyono, Proceedings of the Asia Pacific Industrial Engineering \& Management Systems Conference (2014)

4. T. L. Saaty, Pittsburg: RWS Publications (1988)

5. E. Herrera-Viedma, F. Herrera, F. Chiclana, and M. Luque, European Journal of Operational Research, 154 (2004)

6. T. Tanino, Fuzzy Sets and Systems, 12 (1984)

7. F. Chiclana, F. Herrera, and E. Herrera-Viedma. Fuzzy Sets and Systems. 122 (2001)

8. J. Shanteau, D. J. Weiss, R. P. Thomas, and J. C. Pounds, European Journal of Operational Research, 136 (2002)

9. E. Herrera-Viedma, F. Chiclana, F. Herrera, and S. Alonso, IEEE Transactions on Systems, Man, and Cybernetics (B), 37 (2007)

10. R. R. Yager, IEEE Transactions on Systems, Man, and Cybernetics. 18 (1988)

11. E. Herowati, U. Ciptomulyono, J. Parung, Suparno, Asian Research Publishing Network (ARPN), 9 (2014)

12. F. Chiclana, F. Herrera and E. Herrera-Viedma, Fuzzy Sets and Systems, 97 (1998) 